\title{
Differences of Human Perceptions of a Robot Moving using Linear or Slow in, Slow out Velocity Profiles When Performing a Cleaning Task
}

\author{
Trenton Schulz ${ }^{1}$, Patrick Holthaus ${ }^{2}$, Farshid Amirabdollahian ${ }^{2}$, Kheng Lee Koay ${ }^{2}$, \\ Jim Torresen ${ }^{1}$, and Jo Herstad ${ }^{1}$
}

\begin{abstract}
We investigated how a robot moving with different velocity profiles affects a person's perception of it when working together on a task. The two profiles are the common linear profile and a profile based on the animation principles of slow in, slow out. The investigation was accomplished by running an experiment in a home context where people and the robot cooperated on a clean-up task. We used the Godspeed series of questionnaires to gather people's perception of the robot. Average scores for each series appear not to be different enough to reject the null hypotheses, but looking at the component items provides paths to future areas of research. We also discuss the scenario for the experiment and how it may be used for future research into using animation techniques for moving robots and improving the legibility of a robot's locomotion.
\end{abstract}

\section{INTRODUCTION}

One way older, retired people can live independently at home longer is to have robots in the home to help them with tasks. People interacting with a robot in a home environment will need to perceive the robot as trustworthy, understandable, and something they may want to be around. Can the way a robot speeds up and slows down affect when moving a person's feeling of safety and perception of the robot?

Velocity profiles (or velocity curves) describe the acceleration of a robot. Most robots use a velocity profile that produces linear acceleration (Fig. 1, left), which results in a mechanical, robot-like movement, but there are other velocity profiles available. One of the principles of film animation is the idea of slow in, slow out [1]. In slow in, slow out, the movement starts slowly, but then speeds up to its top speed and slowly stops as it reaches its destination (Fig. 1, right). The goal behind slow in, slow out in film animation is to make a character's movement seem more realistic (that is, like something that is alive and people are familiar with). Applying a slow in, slow out velocity profile to a robot may make the robot's movement more legible and easier to follow resulting in people having a different perception of the robot than if it simply used the linear velocity profile.

In earlier work [2], we implemented an algorithm for changing a ground-based robot's velocity profile to create slow in, slow out movement, but had yet to test how this affects people's perception of a robot, especially when people interact with a robot to complete a task. We wanted to investigate

*Partly funded by the Research Council of Norway as part of the Multimodal Elderly Care Systems (MECS) project, under grant agreement 247697.

${ }^{1}$ Department of Informatics, University of Oslo, Postbox 1080 Blindern, 0316 Oslo [trentonw|jimtoer|johe] difi.uio.no

${ }^{2}$ School of Computer Science, University of Hertfordshire, Hatfield, England [p.holthaus|f.amirabdollahian2 |k.l.koay] @herts.ac.uk
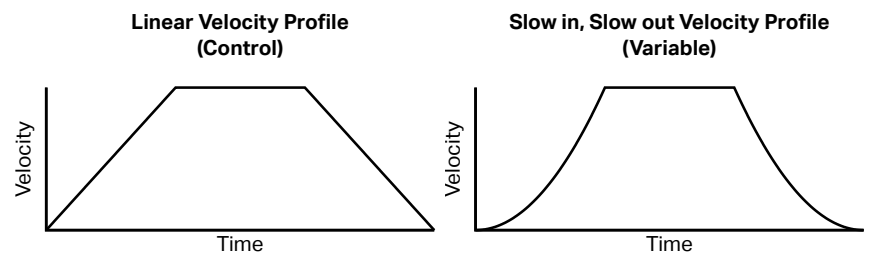

Fig. 1. The experiment tests how people's perception are affected by a robot that moves with a linear velocity profile (left) versus a velocity profile that is based on the animation principle of slow in, slow out (right).

this by running an experiment in a home environment. We previously documented the initial set up for the experiment and the hypotheses we wished to test [3]. Our hypotheses were [3, p. 594]: $(H I)$ a slow in, slow out velocity profile will positively affect the robot's perceived safety versus a linear velocity profile. $(\mathrm{H} 2)$ a slow in, slow out velocity profile will positively affect the robot's perceived intelligence versus a linear velocity profile. $(H 3)$ a slow in, slow out velocity profile will positively affect the robot's perceived animacy versus linear a velocity profile. (H4) a slow in, slow out velocity profile will positively affect the robot's perceived anthropomorphism versus a linear velocity profile. (H5) a slow in, slow out velocity profile will positively affect the robot's perceived likability versus linear a velocity profile. Here, we document the final experimental method (Section III), present results (Section IV), and discussion (Section V). But let us first examine previous human-robot interaction (HRI) research on making robots movement legible and predictable.

\section{RELATED WORK}

Dragan, Lee, and Srinivasa define predictable movement as matching an intent of the moving object; legible movement is where the movement could only lead to the intent of the moving object as observed by a third party [4, p. 301]. One study examined how a robot should approach a person to deliver a drink [5]. People preferred different approaches and distances for the robot-sometimes contradicting preferences from an earlier study [6], but all the participants choose movements emphasizing legibility and a lower risk of harm to the person [5]. This is an example of predictability and legibility for locomotion. Others have attempted to create a typology of meaningful signals and cues for robots based on different modalities to make it easier for people to understand information from a robot they are interacting with [7].

Since a robot's arms can be heavy, powerful, and capable of harm, they are often a focus for legibility studies (legibility 
for configuration). One study found that people familiar with a robot arm's motion makes it easier for them to predict its movement, but it was dependent on how natural the arm's movement was [8]. Another study examined how predictable and legible movement are correlated and formalized the difference between them into a model with the legible motion trying to match natural human grasping motion [4]. This model was applied to a human-robot collaboration study where the legible motion resulted in more fluid collaboration than the predictable motion [9].

Animation principles and techniques have been used make robots' movement easier to understand to people interacting with the robot [10]. For example, a video study used the principles of Anticipation and Follow Through and Overlapping Action to make it easier for viewers to understand what a non-humanoid robot was intending to do [11]. Another study used the timing principle to move a robot's arm, which helped people attribute meaning to the motion [12].

The slow in, slow out principle (also known as easing) has also been used to express intent. This principle was used to help signal to collaborating musicians where a marimbaplaying robot was going to strike [13]. Another study used three principles of animation-anticipation, arcs, and slow in, slow out - to move an assistive free flyer (a type of drone) while performing a task. Participants who watched the drone felt that using these principles made it easier to understand the drone's intent, and the participants also felt that the drone moved naturally and felt safer around the drone [14]. However, newer research indicates that the motion of drones does not directly translate to how terrestrial robots should move [15].

Another study used a new experimental technique where participants viewed frames of a PR2 robot that varied its movement and orientation to see how well participants could predict movement [16]. Participants could spend as much time viewing a frame as they wanted before proceeding to the next. The implication being that the longer the participant looked at the frame, the more time was spent trying to comprehend the robot's movement, and therefore, the less natural and less predictable the movement. The robot moved with four different velocity profiles: linear increasing, linear decreasing, constant, and sinusoidal (vaguely similar to slow in, slow out). Participants spent the most time viewing the frames of a robot with the decreasing linear velocity profile, and on the frames of where the robot decreased speed in the sinusoidal condition, but not when it increased speed. So, the decreasing speed of the robot affected the robot's predictability.

Our work also investigates people's perception of a moving robot and how changing the velocity profile alters that perception. We wanted to investigate this using a real robot in a home context instead of a simulation or a lab setup. We also wanted to focus on a single animation principle to see how much power that principle had on its own to affect people's perception of the robot.

\section{METHOD}

We ran a within-subjects experiment where the variable is how the robot moves: using a linear velocity profile or a slow in, slow out velocity profile. The aim of the experiment was to see if the velocity profiles change people's perception of the robot as they work on a task. We did not want to simply ask people's opinions of the two velocity profiles as people rarely only watch robots move. Therefore, we created a scenario in a home environment that put the focus on the robot interaction, and the robot's movement played a supporting role. We first describe the procedure of the experiment and then provide implementation details about the setting and robot.

\section{A. Experimental Procedure}

The procedure was approved by the University of Hertfordshire Health, Science, Engineering and Technology Ethics Committee (Protocol Number COM/SF/UH/03491). The procedure was as follows. First, a consenting participant entered the home and filled out demographic information of age, gender, and if the participant had any experience with robots. Next, we explained the safety information about the robot for the participant. Then, the scenario was explained: the participant was visiting a friend's house to help in cleaning up the home (one of the facilitators was introduced as the friend). The robot was also helping with the cleaning. Since we did not want to draw attention to robot's motion, we explained we were interested in how the robot handles the hand over of objects from the participant. Then, the participant was given the instructions for what would happen next.

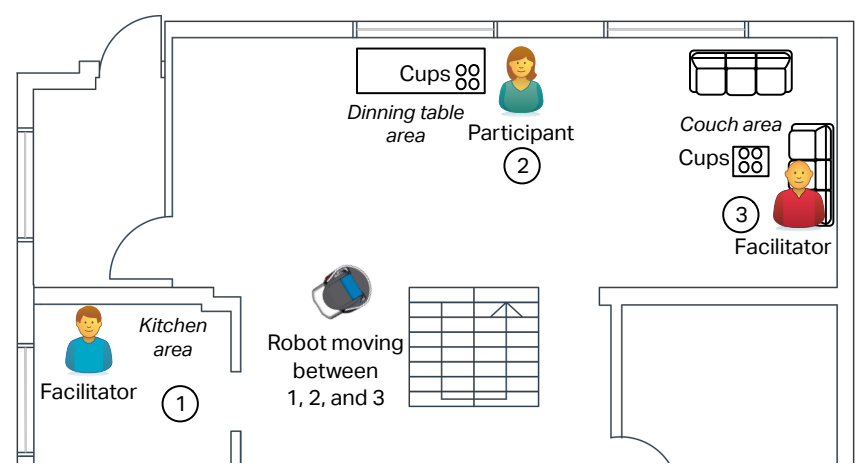

Fig. 2. Floor plan and position of people for the experiment. The robot would move between the numbered positions, starting at Position 1, using a linear or slow in, slow out velocity profile.

The facilitators and participant would then take their positions. This is depicted in Fig. 2. One facilitator would stand in the kitchen (near Position 1 in Fig. 2); the participant and the facilitator helping in cleaning the house would stand near the dining table and sit on the couch respectively (near Positions 2 and 3 respectively in Fig. 2). The dining table and the coffee table by the couch both had four cups on them that would need to be cleaned up.

The robot started in the kitchen (at Position 1 in Fig. 2). The procedure was as follows: (a) The robot moved from Position 1 to Position 2. (b) The participant took one of the cups from the dining table and put it in the robot's basket. (c) The robot moved from Position 2 to Position 3. (d) The facilitator on the couch took a cup from the coffee table and put it in the robot's basket (Fig. 3). (e) The robot moved to 
Position 1. $(f)$ The facilitator in the kitchen removed the cups and put a copy of the questionnaire in the basket. $(g$ ) The robot moved to Position 2. (h) the participant took the questionnaire from the robot and filled it out. (i) Once the questionnaire was complete, the participant put the questionnaire back in the robot's basket. (j) The robot moved to Position 1. (k) Finally, the facilitator in the kitchen removed the questionnaire and prepared the robot for the next iteration.

To measure people's perceptions of the robot, we chose the Godspeed Questionnaire [17], which offers series of semantic scales for measuring a robot's perceived animacy, anthropomorphism, likeability, perceived intelligence, and perceived safety. In addition to these scales, we included an additional item about how well the person could predict where the robot would go, and an open question about how well the person thought the robot performed the task.

This procedure was repeated three times. This resulted in a total of four iterations: two times the movement was with a linear velocity profile, and two times the movement was with a slow in, slow out velocity profile. The profiles were counterbalanced to avoid ordering effects. The counterbalancing was achieved by taking the six possible combinations of two linear and two slow in, slow out velocity profiles, and randomly selecting an ordering for each participant.

Participants were asked to stand if able while giving the cup to the robot and receiving the questionnaire. They could sit while filling out the questionnaire. The primary reason was to allow a better view of Fetch and keep the base for participants' perceptions similar since a standing participant is taller than the robot, which might not be true with a sitting participant. A lesser, secondary reason was to make people feel safer as the robot approached as we reasoned that participants may feel easier to move away from a robot when they are already standing versus having to get up from a chair.

After the final iteration, participants went through an ending procedure where they filled out a questionnaire with openended questions concerning the overall interactions. We also informed participants that we were actually interested in the robot's movement and not the handover. Participants could ask other questions about the experiment and details about the robot and the house. Finally, we thanked participants for their time and, as noted in the informed consent form, gave them a $£ 10$ gift card for Amazon as compensation for time and traveling to Robot House.

\section{B. Experiment Settings, Equipment, and Software}

The home environment was the University of Hertfordshire's Robot House, a house people can visit and experience robots and sensors in a home environment. We used a Fetch Robot (hereafter Fetch) (Fig. 3). Fetch was selected as it can move at a rate of 1 meter per second $(\mathrm{m} / \mathrm{s})$. This speed is slower than an average person's walking speed of $1.4 \mathrm{~m} / \mathrm{s}$ [18], but accelerating up to this speed takes enough time that it is possible to create different velocity profiles. Fetch held a basket to reduce uncertainty in the handover.

Fetch used its own navigation software to navigate to the different points, which is based on the navigation stack from

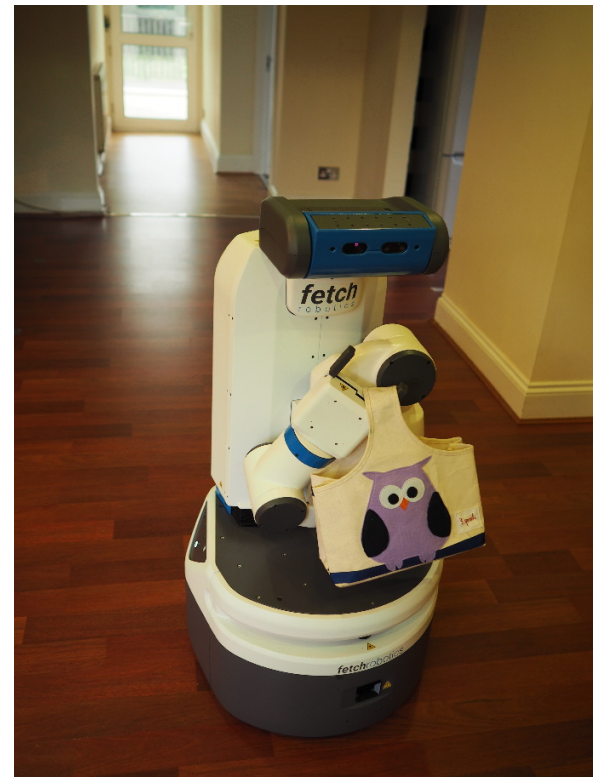

Fig. 3. The Fetch Robot at Robot House, its arm configuration, and the basket used for the experiment.

the Robot Operating System (ROS) [19]. We chose to use the navigation stack as we trusted the stack had better safeguards to move the robot around and avoid obstacles than blind velocity commands. There were pre-assigned destinations to move to in the house: (Positions 1, 2, and 3 respectively on Fig. 2). Each spot had two poses, one for facing the person and one for facing away from the person towards the next location. The two poses per location was done to keep the performance of Fetch's navigation software similar across conditions. Position 1 had a slightly different locations for its poses to make it easier to remove and add items to the basket without the participant noticing.

In an effort to improve the internal validity of the experiment, we did not want confounding factors such as speech recognition or sound to be part of the experiment. We only wanted to use motion. When Fetch had arrived at the pose facing the person, it would raise its torso $10 \mathrm{~cm}$. to indicate that it was ready to receive a cup, or that the person should take (and later return) the questionnaire. This movement—one could argue a form of configuration legibility — was added after a pilot study showed that a participant could be confused as to when the participant should give the cup to the robot.

For the velocity profiles, we adapted the algorithm from Schulz, Herstad, and Torresen [2] to be a plugin for the local planner in the navigation system. The plugin was a modified version of Fetch's local planner and was based on the trajectory roll out scheme [20]. This was similar to a suggested set up for integrating stylized motion into a velocity profile for a task [21]. The plugin included an additional parameter that can be modified dynamically for setting the velocity profile (linear or slow in, slow out). This allowed us to quickly change the velocity profile without having to restart the robot's navigation system. The changes only affected Fetch's linear velocity (i.e., moving forward); 
the angular velocity (i.e., turning in place) was always based on a linear velocity profile.

Fetch was partially controlled via Wizard of Oz. The Wizard of $\mathrm{Oz}$ component consisted of the facilitator in the kitchen (the paper's first author) watching when the participant and the facilitator on the couch had put a cup in Fetch's basket, and then instructing Fetch to navigate to the next Position. This allowed us to reduce time variability of the experiment; we also did not have time to implement adding sensors and code for checking that the cups had been put in the basket. The choice of having a person in the kitchen and the couch also allowed two people to watch the robot and activate an emergency stop if Fetch was going to run into something.

We collected additional data from Fetch. This included the robot's odometry information (e.g., Fig. 4) and the time from when a request to move was made to move to the next location until the time that the robot arrived at the location and raised its torso.

\section{RESULTS}

There were 38 participants who performed the experiment. 19 of the participants identified as female and 19 as male. Ages range from 18 to 80 years (mean age: 37.39 years, median age: 34.5 years, SD: 15.74 years). 16 participants had no experience with robots. Each participant had two interactions with the linear velocity profile and two interactions with the slow in, slow out profile for a total of 152 encounters (76 linear and 76 slow in, slow out).

We collected quantitative and qualitative data, but due to space constraints, we will only present the Godspeed series results. Since the Godspeed Perceived Safety has two items where the negative item is at the top of the scale and the positive item at the bottom of the scale, we have reversed those items for the calculations presented below.

\section{A. Tests for Data Quality}

The main measure was the Godspeed series. In introducing the series, practitioners were advised to use Cronbach's $\alpha$ to test for the internal consistency of the questions and to see if the responses were reliable for each series of questions [17]. The results are in Table I.

TABLE I. Cronbach's $\alpha$ for each Godspeed series

\begin{tabular}{lc}
\hline Godspeed series & Cronbach's $\alpha$ \\
\hline Anthropomorphism & 0.86 \\
Animacy & 0.84 \\
Likeability & 0.9 \\
Perceived Intelligence & 0.84 \\
Perceived Safety & 0.63 \\
\hline
\end{tabular}

The rule of thumb is a Cronbach's $\alpha$ over 0.7 has good internal consistency. The Anthropomorphism, Animacy, Likeability, and Perceived Intelligence series exceed this threshold and should have good consistency (Table I). The Perceived Safety series is below the 0.7 threshold at 0.63. This series has fewer items than the other series (three versus five or more for the others). However, the score indicates a fair agreement among the perceived safety variables. So, the Godspeed Perceived Safety was still considered below.

We then ran a Shapiro-Wilk normality test to see if the responses were a normal distribution. The test was run on all the Godspeed semantic items, and results indicated the data was not a normal distribution (all $p<.0000001$ ). However, running the test on the average of items per series indicated that the average for Anthropomorphism series may be a normal distribution $(p>.05)$. Regardless, the Shapiro-Wilk test indicated that we should employ statistical tests that did not assume a normal distribution like the Wilcoxon MatchedPairs Signed-Ranks test when checking statistical significance, and bootstrapping when calculating confidence intervals (CI).

\section{B. Hypothesis Testing}

TABLE II. Number of participants with complete responses, actual and bootstrapped mean, and Bootstrap Bias-Corrected Accelerated 95\% Confidence interval for Godspeed series used in experiment, split by Linear and Slow in, Slow out velocity profile.

\begin{tabular}{lcccc}
\hline Godspeed series & $n$ & Mean & Bootstrap & 95\% BCa CI \\
\hline Anthropomorphism & & & & \\
Linear & 36 & 2.84 & 2.84 & $(2.56,3.06)$ \\
Slow in, Slow out & 37 & 2.83 & 2.83 & $(2.60,3.08)$ \\
\hline Animacy & & & & \\
Linear & 37 & 2.88 & 2.88 & $(2.65,3.1)$ \\
Slow in, Slow out & 38 & 2.87 & 2.87 & $(2.66,3.1)$ \\
Likeability & & & & \\
Linear & 37 & 3.59 & 3.59 & $(3.37,3.82)$ \\
Slow in, Slow out & 38 & 3.71 & 3.71 & $(3.52,3.92)$ \\
\hline Perceived Intelligence & & & & \\
Linear & 37 & 3.57 & 3.57 & $(3.36,3.77)$ \\
Slow in, Slow out & 37 & 3.51 & 3.51 & $(3.32,3.69)$ \\
\hline Perceived Safety & & & & \\
Linear & 38 & 3.93 & 3.93 & $(3.71,4.12)$ \\
Slow in, Slow out & 38 & 3.98 & 3.98 & $(3.77,4.19)$ \\
\hline
\end{tabular}

The corresponding null hypotheses for the hypotheses in Section I would be there is no difference between the perceived qualities of the robot and velocity profiles. We grouped each participants' encounters by linear and slow in, slow out velocity profile and calculated the average (mean) score for each semantic item in these groups; missing responses to one or more missing semantic items were dropped for that series (i.e., $n=38$ for groups with no missing items). We then took the mean of all items for each series. Since this data is not normally distributed, we used bootstrapping with 2,000 iterations to calculate a $95 \%$ Bias-Corrected accelerated (BCa) confidence interval on the average. The series' distribution is presented as a box plot (Fig. 5). The mean scores and CIs are presented in Table II.

Table II shows small differences in the means and large overlap in the CIs. Fig. 5 shows differences in terms of the extremes and the grouping for likeability and perceived intelligence, but is there a statistically significant difference? Wilcoxon Matched-Pairs Signed-Ranks tests were run on the means to see if we could reject the null hypotheses. Since the hypotheses are comparisons in a family, we ran a HolmBonferroni correction to check that multiple comparisons do 

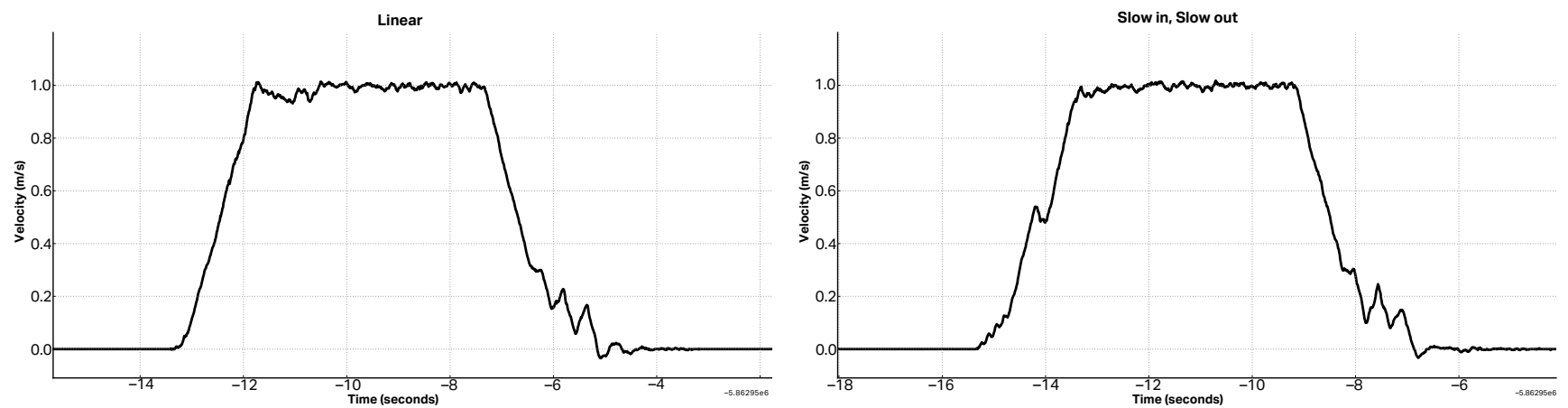

Fig. 4. Odometry samples of the robot's linear velocity when moving from Position 3 to Position 1 for linear (left) and slow in, slow out (right). Data line has been thickened for readability at this size. The time spent moving from start to finish is similar.

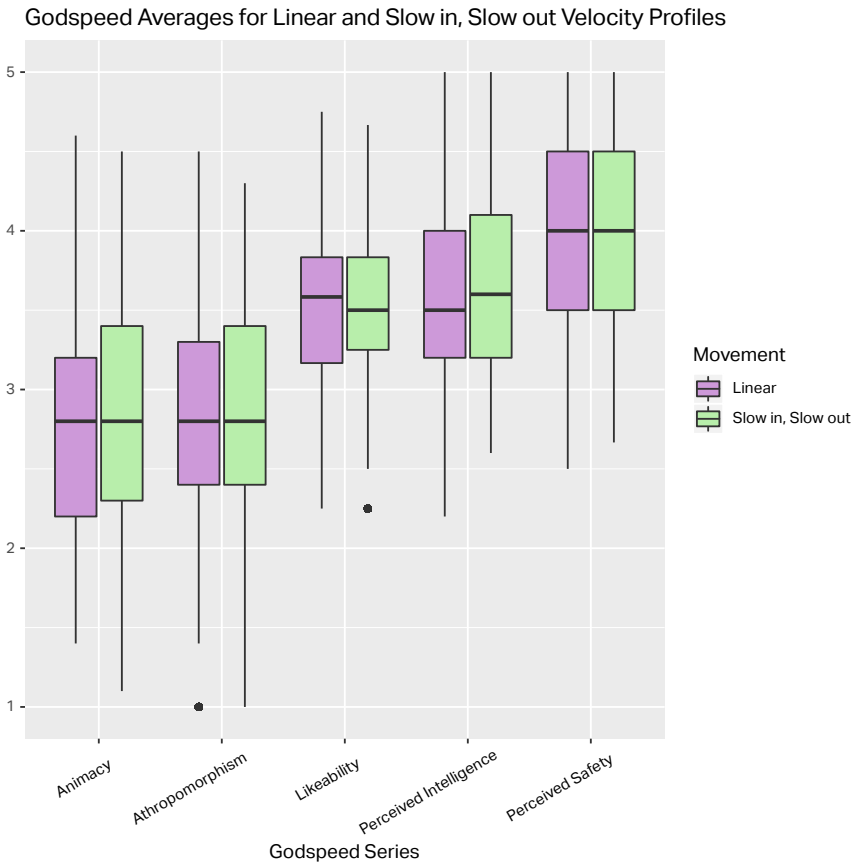

Fig. 5. Box plot of the averages for each Godspeed Series, split by Slow in, Slow out and Linear.

not result in something randomly becoming significant. The Holm-Bonferroni correction indicated none of the groupings were statistically significant (all $p>.05$ ). This, combined with the CIs, indicate we cannot reject the null hypotheses. These results were not appreciably different when only looking at participants who had no experience with robots.

\section{DISCUSSION}

Altering a velocity profile can have an effect on the safety of the robot. We expected to see a pronounced effect between the velocity profiles, but this was not the case. However, presenting these results are important for other HRI researchers to know that a certain technique does not provide a certain effect. Using animation techniques with robots are under investigation and used in other studies as presented in Section II, and will likely continue to do so. Knowing what works or what does not work helps this research.
This also provides an opportunity to help in the design of future studies. One place to look for future research directions is to review responses to the different semantic items of the Godspeed Series. We can also reexamine the experiment to see if there are other items that need to be considered.

\section{A. Semantic Item Exploration}

Although we could not reject the null hypotheses, we explored the semantic items for cases where there might be differences. As this study was not specifically looking at these comparisons, we do not draw conclusions, but they may point to possible items to explore in future studies.

We ran Wilcoxon Matched-Pairs Signed-Ranks tests and bootstrapped $95 \%$ confidence intervals on the semantic items. This showed three interesting items: the Unpredictable-Predictable item for the Perceived Intelligence Series (Fig. 6, Table III), the Inert-Interactive item for the Animacy series (Fig. 7, Table IV), and the Calm-Agitated item for the Perceived Safety series (Fig. 8, Table V).

TABLE III. Participants with complete responses, actual and bootstrapped mean, and 95\% BCa CI for Godspeed Perceived Intelligence semantic items used in experiment, split by Linear and Slow in, Slow out velocity profile.

\begin{tabular}{lrccc}
\hline $\begin{array}{l}\text { Perceived Intelligence } \\
\text { Item }\end{array}$ & $n$ & Mean & Bootstrap & 95\% BCa CI \\
\hline $\begin{array}{l}\text { Incompetent-Competent } \\
\text { Linear }\end{array}$ & 37 & 3.84 & 3.84 & $(3.55,4.04)$ \\
Slow in, Slow out & 37 & 3.78 & 3.78 & $(3.58,3.96)$ \\
\hline $\begin{array}{l}\text { Ignorant-Knowledgeable } \\
\text { Linear }\end{array}$ & 38 & 3.28 & 3.28 & $(3.00,3.54)$ \\
Slow in, Slow out & 38 & 3.33 & 3.32 & $(3.09,3.55)$ \\
\hline $\begin{array}{l}\text { Irresponsible-Responsible } \\
\text { Linear }\end{array}$ & 38 & 3.55 & 3.55 & $(3.38,3.88)$ \\
Slow in, Slow out & 38 & 3.63 & 3.63 & $(3.32,3.79)$ \\
\hline $\begin{array}{l}\text { Unintelligent-Intelligent } \\
\text { Linear }\end{array}$ & 38 & 3.39 & 3.40 & $(3.09,3.67)$ \\
Slow in, Slow out & 38 & 3.30 & 3.31 & $(3.00,3.59)$ \\
\hline $\begin{array}{l}\text { Foolish-Sensible } \\
\text { Linear }\end{array}$ & 38 & 3.47 & 3.47 & $(3.19,3.74)$ \\
Slow in, Slow out & 38 & 3.38 & 3.38 & $(3.13,3.61)$ \\
\hline Unpredictable-Predictable & & & \\
Linear & 38 & 3.96 & 3.96 & $(3.71,4.16)$ \\
Slow in, Slow out & 38 & 3.70 & 3.70 & $(3.42,3.89)$ \\
\hline
\end{tabular}




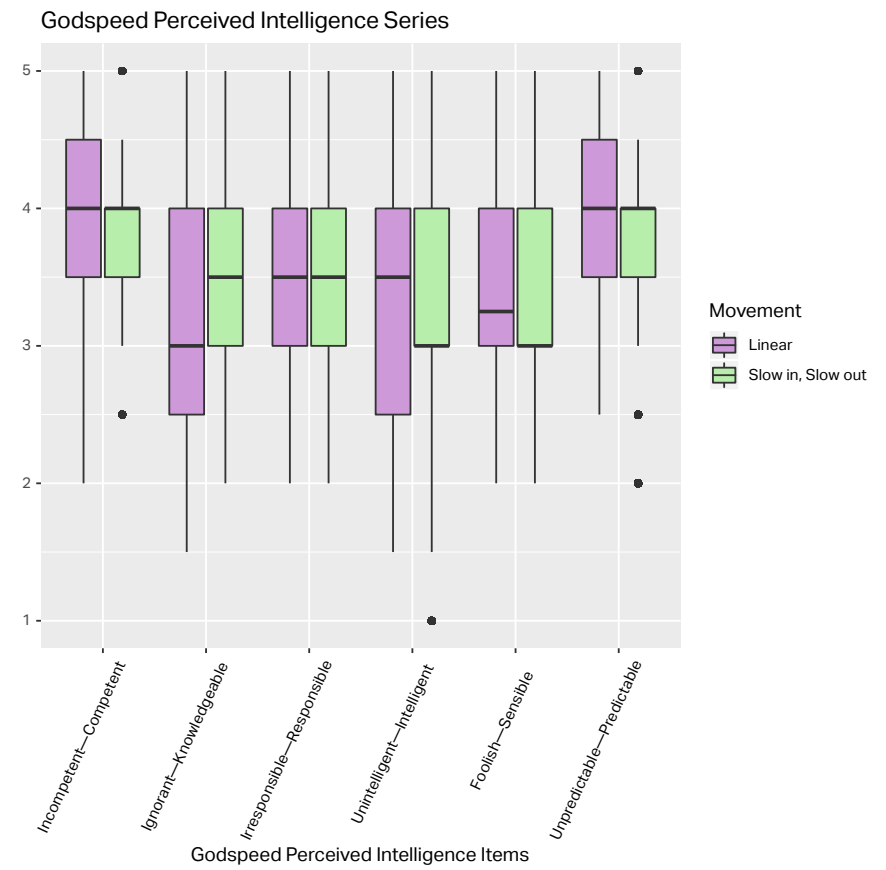

Fig. 6. Box plot of the averages the Perceived Intelligence semantic items, split by Slow in, Slow out and Linear.

TABLE IV. Participants with complete responses, actual and bootstrapped mean, and 95\% BCa CI for Godspeed Animacy semantic items used in experiment, split by Linear and Slow in, Slow out velocity profile; the participants who received the wrong Inert-Interactive item are removed.

\begin{tabular}{lcccc}
\hline Animacy Item & $n$ & Mean & Bootstrap & 95\% BCa CI \\
\hline Dead-Alive & & & & \\
Linear & 31 & 3.18 & 3.18 & $(2.77,3.53)$ \\
Slow in, Slow out & 32 & 3.19 & 3.19 & $(2.83,3.50)$ \\
\hline Stagnant-Lively & & & & \\
Linear & 31 & 3.27 & 3.28 & $(2.95,3.56)$ \\
Slow in, Slow out & 32 & 3.23 & 3.24 & $(2.91,3.50)$ \\
\hline Mechanical-Organic & & & & \\
Linear & 31 & 2.34 & 2.34 & $(2.15,3.66)$ \\
Slow in, Slow out & 32 & 2.41 & 2.41 & $(2.15,2.65)$ \\
\hline Artificial-Lifelike & & & & \\
Linear & 31 & 2.52 & 2.51 & $(2.21,2.79)$ \\
Slow in, Slow out & 32 & 2.52 & 2.52 & $(2.23,2.81)$ \\
\hline Inert-Interactive & & & & \\
Linear & 31 & 3.13 & 3.13 & $(2.87,3.39)$ \\
Slow in, Slow out & 32 & 3.03 & 3.03 & $(2.67,3.38)$ \\
\hline
\end{tabular}

Looking first at the Unpredictable-Predictable series, we suspect there was a confounding factor that caused the difference in being unpredictable. When preparing the experiment, we found no noticeable difference in the time for the robot to move between positions. Fig. 4 shows the times for Fetch to travel from Position 3 to Position 1 are within a half second between velocity profiles.

However, during the experiment the averages of the timings we recorded shows a large difference between instructing the robot to move from Position 3 to Position 1 and the robot completing the move. The time the robot used in preparing for this move sometimes took much longer (on average about

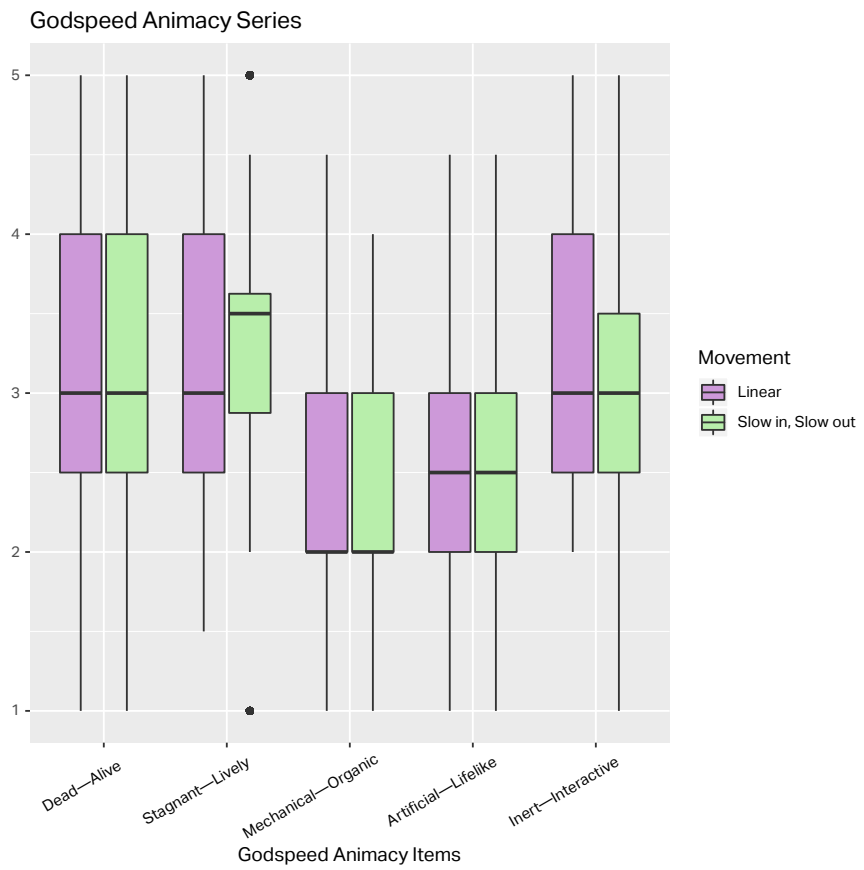

Fig. 7. Box plot of averages of Animacy semantic items; the participants who received the wrong Inert-Interactive item are removed.

TABLE V. Participants with complete responses, actual and bootstrapped mean, and 95\% BCa CI for Godspeed Perceived Safety semantic items used in experiment, split by Linear and Slow in, Slow out velocity profile.

\begin{tabular}{lcccc}
\hline Perceived Safety Item & $n$ & Mean & Bootstrap & 95\% BCa CI \\
\hline Anxious-Relaxed & & & & \\
Linear & 38 & 4.20 & 4.20 & $(3.93,4.42)$ \\
Slow in, Slow out & 38 & 4.07 & 4.06 & $(3.69,4.33)$ \\
\hline Agitated-Calm & & & & \\
Linear & 38 & 4.12 & 4.11 & $(3.74,4.38)$ \\
Slow in, Slow out & 38 & 4.30 & 4.30 & $(4.03,4.51)$ \\
\hline Surprised-Quiescent & & & & \\
Linear & 38 & 3.47 & 3.47 & $(3.18,3.71)$ \\
Slow in, Slow out & 38 & 3.57 & 3.56 & $(3.30,3.80)$ \\
\hline
\end{tabular}

five seconds longer for the slow in, slow out velocity profile). In these situations, Fetch's navigation system would register Fetch as "stuck" and rotate the robot to help reset its local obstacle map. This would add more time from when the robot was requested to move until it completed its move. This happened more often for the slow in, slow out condition than the linear condition (17 out of 76 times versus 4 out of 76 times) and may explain the difference. Many participants noted this rotating when filling out the questionnaires and this could also have resulted in the perception in predictability. We are unsure what caused this "stuck" behavior. We suspect that it was latency with the wireless network combined with delays in the navigation system being run on a different computer and not Fetch. This did not turn up in our pilot, but we should have accounted for the possibility of this behavior.

Examining the Intert-Interactive semantic item for the Animacy series had problems as its semantic scale was incorrect for 6 participants (Due to a typo, the scale was Inert-Inactive 


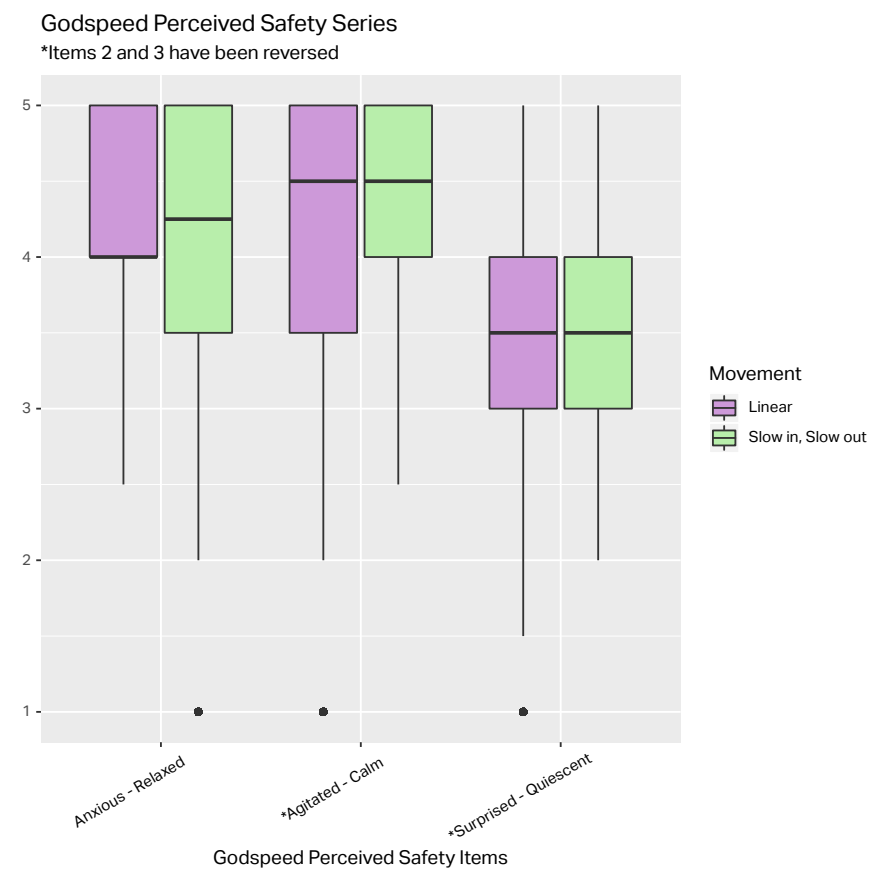

Fig. 8. Box plot of the averages the Perceived Safety semantic items, split by Slow in, Slow out and Linear.

instead of Inert-Interactive). This was corrected for the other 32 participants. When these 6 participants were removed, the resulting test shows no statistically significant difference.

The box plot for the Perceived Safety series (Fig. 8) shows more concentration at the calm end of the semantic item for the slow in, slow out versus the linear velocity profile, and Table $\mathrm{V}$ indicates the average may be different. However, the Wilcoxon Signed Ranks test is a test of how the spread of the scores match, not the match in average score. Figs. 6-8 show that the median scores of Distracted-Calm, Unpredictable-Predictable, and Inert-Interactive semantic items are the same between the Linear and Slow in, Slow out velocity profiles. Future work would need to examine whether the concentration is real and desirable. Other items did not show a statistically significant difference and had similar averages, but had different medians (e.g., Stagnant-Lively of the Animacy Series in Fig.7 and Table IV).

\section{B. Examining the Scenario}

The scenario we described can be used by others also wishing to examine aspects of a robot-like movement-while the person is engaged in a task with the robot in a home context. Participants in the experiment generally watched the robot most of the time it was moving, so this might be useful for studies requiring participants to watch a robot.

Most animation work consists of several principles. One reason we ran this study was to see how powerful one principle of animation on its own could be in a scenario. That is, if we only employ one principle for a robot in a real world scenario, does it make a difference in people's perceptions? In this scenario, the slow in, slow out velocity profile is not enough to change perceptions of animacy, anthropomorphism, likeability, perceived intelligence, or perceived safety on its own. But there might be other factors that are overpowering the principle. For example, the movement of the robot's torso and robot's turning did not use slow in, slow out. This may mean that these other velocity profiles overpowered the robot's movement from the different positions. In addition, Fetch's momentum also created a type of slow in, slow out effect even in the linear velocity profile (visible in Fig. 4). Events such as the robot getting "stuck" may also have a larger impact than the slow in, slow out velocity profile on its own. Even other properties of Fetch-such as appearance, color, or texture appearance may overpower how it moves. So this raises a question about how much the result is tied to the specific robot for the scenario.

As mentioned in Section III, people will likely be interacting with a robot, not simply watching how it moves. The slow in, slow out velocity profile may be too subtle on its own for people to easily perceive the difference while working on the task. Perhaps changes can be made to the velocity curves to make them more distinct, fixing the "stuck" events, a different approach angle, or a different activity could make the differences easier to notice.

It may also be that animation principles must be combined to get a noticeable effect. Participants in a study of a greeting robot that used a straight movement versus movement based on the animation principle of arcs did not mention any difference between these two types of movements in their interviews [22]. Further research is needed to see how combining principles can lead to noticeable effects and if the noticeable effects are desired in a robot's design.

Another issue can be that Godspeed questionnaire may not be the best tool to capture the perceptions here. Our exploration of the semantic items suggest examining safety, legibility, and predictability with the slow in, slow out velocity profile. A different measurement tool, perhaps based on the perceived safety scale, may provide a better answer in a future version of this scenario. On the other hand, others have suggested that a subjective measurement scale may not be sufficient for capturing people's perceptions, and the results could differ from observed data [23]. Qualitative data may also provide a richer picture in these circumstances. We are examining the qualitative data from our participants and will present this in the future. Different tools and a study focusing on one element may provide stronger statistical power on the quantitative side, and a deeper understanding on the phenomena on the qualitative side.

This set up also shows the need to carefully decide hypotheses for quantitative measures. Adding a measure (here, an additional Godspeed series) broadens the scope of what you can find, but it adds additional requirements on rejecting a null hypothesis. It is important to consider using descriptive statistics to help explain the effect the data is showing [24].

\section{CONCLUSION AND FUTURE WORK}

We ran a within-subjects survey looking at how a person perceives a robot moving with a linear velocity profile versus a profile based on the slow in, slow out animation 
principle, while performing a task together. We used the Godspeed Questionnaire series to measure the perceptions of anthropomorphism, animacy, likeability, perceived intelligence, and perceived safety. The average scores of these series indicate that there is not enough difference to indicate an effect. We have documented our study and provided the implementation for the velocity curves, the software for running the experiment, and for analyzing results ${ }^{1}$. We are examining the qualitative data to see if we can create a more complete picture of people's perceptions.

Even without any major perceived distinction between the two profiles, there are still possibilities to explore with the slow in, slow out velocity profile. Examining the Godspeed semantic items shows that aspects of participants' perceived safety may be influenced with a slow in, slow out velocity profile. Future work could examine if a slow in, slow out velocity profile provides a better indication of a robot starting and stopping and thus makes the robot's locomotion more legible or predictable to the humans interacting with it. Another aspect would be combining slow in, slow out with other principles like anticipation to further enhance this legibility. A focused study on these topics, supplemented with other research methods, can provide insight into how humans and robots can work together safely.

\section{ACKNOWLEDGMENT}

We thank Robot House at the University of Hertfordshire for allowing us to use their facilities to design and run the experiment described in this paper. We also thank Ragnar Hauge for suggestions and help on statistical methods.

\section{REFERENCES}

[1] F. Thomas and O. Johnston, The Illusion of Life: Disney Animation, 1st Hyperion ed. New York: Hyperion, 1995, 575 pp.

[2] T. Schulz, J. Herstad, and J. Torresen, "Classifying Human and Robot Movement at Home and Implementing Robot Movement Using the Slow In, Slow Out Animation Principle," International Journal on Advances in Intelligent Systems, vol. 11, pp. 234-244, 3 \& 42018.

[3] T. Schulz, P. Holthaus, F. Amirabdollahian, and K. L. Koay, "Humans' Perception of a Robot Moving Using a Slow in and Slow Out Velocity Profile," in 2019 14th ACM/IEEE International Conference on Human-Robot Interaction (HRI), 2019, pp. 594-595. DOI: $10.1109 / \mathrm{HRI} .2019 .8673239$.

[4] A. D. Dragan, K. C. Lee, and S. S. Srinivasa, "Legibility and Predictability of Robot Motion," in Proceedings of the 8th ACM/IEEE International Conference on Human-Robot Interaction, ser. HRI '13, Piscataway, NJ, USA: IEEE Press, 2013, pp. 301-308.

[5] K. Koay, E. A. Sisbot, D. Syrdal, et al., "Exploratory Study of a Robot Approaching a Person in the Context of Handing Over an Object," presented at the AAAI Spring Symposium: Multidisciplinary Collaboration for Socially Assistive Robotics, Stanford, California, USA, 2007.

[6] M. L. Walters, K. Dautenhahn, S. N. Woods, and K. L. Koay, "Robotic Etiquette: Results from User Studies Involving a Fetch and Carry Task," in Proceedings of the ACM/IEEE International Conference on Human-Robot Interaction, ser. HRI '07, New York, NY, USA: ACM, 2007, pp. 317-324. DOI: 10.1145/1228716. 1228759.

[7] F. Hegel, S. Gieselmann, A. Peters, P. Holthaus, and B. Wrede, "Towards a typology of meaningful signals and cues in social robotics," in 2011 20th IEEE International Symposium on Robot and Human Interactive Communication (RO-MAN), IEEE, 2011, pp. 72-78.
[8] A. Dragan and S. Srinivasa, "Familiarization to Robot Motion," in Proceedings of the 2014 ACM/IEEE International Conference on Human-Robot Interaction, ser. HRI '14, New York, NY, USA: ACM, 2014, pp. 366-373. DOI: 10.1145/2559636.2559674.

[9] A. D. Dragan, S. Bauman, J. Forlizzi, and S. S. Srinivasa, "Effects of Robot Motion on Human-Robot Collaboration," in Proceedings of the Tenth Annual ACM/IEEE International Conference on HumanRobot Interaction, ser. HRI '15, New York, NY, USA: ACM, 2015, pp. 51-58. DOI: $10.1145 / 2696454.2696473$.

[10] T. Schulz, J. Torresen, and J. Herstad, "Animation Techniques in Human-Robot Interaction User Studies: A Systematic Literature Review," ACM Transactions on Human-Robot Interaction, vol. 8, no. 2, 2019. DOI: 10.1145/3317325.

[11] L. Takayama, D. Dooley, and W. Ju, "Expressing Thought: Improving Robot Readability with Animation Principles," in Proceedings of the 6th International Conference on Human-Robot Interaction, ser. HRI '11, New York, NY, USA: ACM, 2011, pp. 69-76. D OI: $10.1145 /$ 1957656.1957674 .

[12] A. Zhou, D. Hadfield-Menell, A. Nagabandi, and A. D. Dragan, "Expressive Robot Motion Timing," in Proceedings of the 2017 ACM/IEEE International Conference on Human-Robot Interaction, ser. HRI '17, New York, NY, USA: ACM, 2017, pp. 22-31. D OI: $10.1145 / 2909824.3020221$.

[13] G. Hoffman and G. Weinberg, "Interactive improvisation with a robotic marimba player," Autonomous Robots, vol. 31, no. 2-3, pp. 133-153, 2011, cited By 18. DOI: 10.1007/s10514-0119237-0.

[14] D. Szafir, B. Mutlu, and T. Fong, "Communication of Intent in Assistive Free Flyers," in Proceedings of the 2014 ACM/IEEE International Conference on Human-Robot Interaction, ser. HRI '14, New York, NY, USA: ACM, 2014, pp. 358-365. D OI: 10 . $1145 / 2559636.2559672$.

[15] A. Wojciechowska, J. Frey, S. Sass, R. Shafir, and J. R. Cauchard, "Collocated Human-Drone Interaction: Methodology and Approach Strategy," in Proceedings of the 14th ACM/IEEE Conference on Human-Robot Interaction (HRI '19), IEEE, 2019, pp. 172-181.

[16] F. Papenmeier, M. Uhrig, and A. Kirsch, "Human Understanding of Robot Motion: The Role of Velocity and Orientation," Int J of Soc Robotics, 2018. DOI: 10.1007/s12369-018-0493-4.

[17] C. Bartneck, D. Kulić, E. Croft, and S. Zoghbi, "Measurement Instruments for the Anthropomorphism, Animacy, Likeability, Perceived Intelligence, and Perceived Safety of Robots," International Journal of Social Robotics, vol. 1, no. 1, pp. 71-81, 2009. D OI: $10.1007 /$ s12369-008-0001-3.

[18] J. Rose, D. W. Morgan, and J. G. Gamble, "Energetics of Walking," in Human Walking, J. Rose and J. G. Gamble, Eds., 3rd, Philadelphia, PA, USA: Lippincott Williams \& Wilkins, 2006, pp. 77-102.

[19] Open Source Robotics Foundation. (2019). About ROS, [Online]. Available: http://www.ros.org/about-ros/ (visited on 2019-04-03)

[20] B. P. Gerkey and K. Konolige, "Planning and control in unstructured terrain," in In Workshop on Path Planning on Costmaps, Proceedings of the IEEE International Conference on Robotics and Automation (ICRA), 2008.

[21] M. J. Gielniak, C. K. Liu, and A. Thomaz, "Stylized motion generalization through adaptation of velocity profiles," in $2010 \mathrm{RO}$ MAN: The 19th IEEE International Symposium on Robot and Human Interactive Communication, IEEE, 2010, pp. 304-309.

[22] L. Anderson-Bashan, B. Megidish, H. Erel, et al., "The Greeting Machine: An Abstract Robotic Object for Opening Encounters," in 2018 27th IEEE International Symposium on Robot and Human Interactive Communication (RO-MAN), 2018, pp. 595-602. D OI: 10.1109/ROMAN.2018.8525516.

[23] K. Winkle, S. Lemaignan, P. Caleb-Solly, et al., "Effective Persuasion Strategies for Socially Assistive Robots," in 2019 14th ACM/IEEE International Conference on Human-Robot Interaction (HRI), 2019, pp. 277-285. DOI: 10.1109/HRI.2019.8673313.

[24] P. Baxter, J. Kennedy, E. Senft, S. Lemaignan, and T. Belpaeme, "From characterising three years of HRI to methodology and reporting recommendations," in 2016 11th ACM/IEEE International Conference on Human-Robot Interaction (HRI), 2016, pp. 391-398. DOI: 10.1109/HRI.2016.7451777. 